\title{
Correction to: Customer Experience and Its Marketing Outcomes in Financial Services: A Multivariate Approach
}

\author{
Swati Raina, Hardeep Chahal and Kamani Dutta
}

\section{Correction to:}

Chapter 7 in: H. Chahal et al. (eds.), Understanding the Role of Business Analytics, https://doi.org/10.1007/978-981-13-1334-9_7

In the original version of this chapter, the author Phillip Klaus' name was included erroneously as a co-author. This has now been rectified and the author name has been removed. 